\title{
Nosocomial transmission of chickenpox and varicella zoster virus seroprevalence rate amongst healthcare workers in a teaching hospital in China
}

Jin Yang ${ }^{1}$, Jieling Liu', Fanfan Xing ${ }^{1}$, Haiyan Ye ${ }^{1}$, Guijian Dai ${ }^{1}$, Meiyuan Liu', Simon Kam-Fai Lo', Ricky Wing-Tong Lau', Kelvin Hei-Yeung Chiu' ${ }^{3}$, Jasper Fuk-Woo Chan ${ }^{1,2,3,4}$ and Kwok-Yung Yuen ${ }^{1,2,3,4,5^{*}}$

\begin{abstract}
Background: Varicella zoster virus (VZV) is a highly contagious herpesvirus with potential for nosocomial transmission. However, the importance of nosocomial chickenpox outbreak in China has often been ignored. With the increasing immunocompromised population in China, a thorough review of issues related to nosocomial transmission and the seroprevalence rate of VZV among healthcare workers is necessary.

Methods: Retrospective case finding for nosocomial transmission of chickenpox was conducted between January 1 , 2013 and December 31, 2017. Cases were identified based on clinical features compatible with chickenpox. A cross-sectional study on the seroprevalence rate of VZV among healthcare workers (HCWs) was conducted between January 1, 2014 and December 31, 2017. The serum VZV antibodies of 1804 HCWs were measured by enzyme-linked immunosorbent assay (ELISA). The seroprevalence rate of VZV antibodies, the positive predictive value and negative predictive value of self-reported history of varicella were analyzed. The economic impact associated with nosocomial transmission of VZV was also assessed.

Results: A total of 8 cases of chickenpox were identified in three nosocomial transmissions, including 4 HCWs who were infected nosocomially. The overall seroprevalence rate of VZV was $88.4 \%$, which significantly increased with age $(P<0.01)$. The seroprevalence rates of HCWs with different genders and occupations showed no statistically significant differences. The positive and negative predictive values of a self-reported history of varicella were 80.8 and $10.6 \%$ respectively. An estimation of 163.3 person-days of work were lost in each nosocomial transmission and 86.7 infection control unit person-hours were required for each outbreak investigation. The cost of VZV IgG ELISA screening was estimated to be 83 USD per nosocomial transmission.

Conclusions: Nosocomial transmission of VZV occurred repeatedly in the hospital setting. An alarming $11.6 \%$ of HCWs were seronegative for VZV, which might increase the risk of nosocomial infection and outbreak for other susceptible co-workers and patients. This is especially important in the setting of a teaching hospital where many immunocompromised patients were managed. Furthermore, the positive predictive value of self-reported varicella on seroprevalence rate in our study was lower than those reported in other countries, therefore serological testing of VZV antibodies with subsequent vaccination for all non-immune HCWs should be considered.
\end{abstract}

Keywords: Chickenpox, China, Healthcare worker, Outbreak, Seroprevalence, Varicella zoster virus

\footnotetext{
* Correspondence: kyyuen@hku.hk

'Department of Clinical Microbiology and Infection Control, The University of

Hong Kong - Shenzhen Hospital, Shenzhen, China

${ }^{2}$ State Key Laboratory of Emerging Infectious Diseases, The University of

Hong Kong, Pokfulam, Hong Kong, Special Administrative Region of China

Full list of author information is available at the end of the article
}

(c) The Author(s). 2019 Open Access This article is distributed under the terms of the Creative Commons Attribution 4.0 International License (http://creativecommons.org/licenses/by/4.0/), which permits unrestricted use, distribution, and reproduction in any medium, provided you give appropriate credit to the original author(s) and the source, provide a link to the Creative Commons license, and indicate if changes were made. The Creative Commons Public Domain Dedication waiver (http://creativecommons.org/publicdomain/zero/1.0/) applies to the data made available in this article, unless otherwise stated. 


\section{Background}

Varicella zoster virus (VZV) causes two clinically distinct forms of disease: varicella (chickenpox), as a primary infection, and zoster (shingles) due to reactivation of latent VZV [1-3]. The transmission of VZV occurs via inhalation of airborne droplets or direct contact with vesicular fluid from skin lesions. VZV infection is considered an occupational hazard for susceptible healthcare workers (HCWs), as it can spread to other susceptible coworkers and patients [4]. Transmission of VZV among HCWs and patients has been reported [5-7]. Current policy in China for prevention of nosocomial transmission of VZV is prompt recognition of patients with suspected VZV infection with airborne isolation in negative pressure single room if possible, however mandatory VZV IgG testing is still not yet implemented. Thus HCWs with negative history of chickenpox and VZV vaccination should be screened for VZV antibodies. Immune status to VZV can be assessed by enzyme-linked immunosorbent assays (ELISAs), or fluorescent antibody to membrane antigen (FAMA) assays [8]. Seronegative HCWs are considered susceptible to varicella, therefore VZV vaccination is recommended [4].

Primary VZV infection is usually self-limiting in immunocompetent hosts, however, the severity increases with age. Furthermore, as HCWs care for patients in different wards and units, there is a high potential of nosocomial transmission among HCWs and patients if the HCW is infected, especially when the infectious period of VZV starts 2 days before the onset of rash. The situation would be worse if the infected HCW is involved in taking care of pregnant women and immunocompromised individuals (such as patients receiving high dose immunosuppressant or history of transplantation), as primary VZV infection in immunocompromised patients can be fulminant, and it can present as encephalitis, pneumonitis and hepatitis. The mortality rate of primary VZV infection could be up to $7 \%$ if left untreated. As a result, nosocomial transmission of VZV should never be overlooked.

As nosocomial transmission of varicella is seldom reported in China, this study aims to report three nosocomial transmissions of chickenpox, and investigate the seroprevalence rate of VZV among healthcare workers in a tertiary teaching hospital in China.

\section{Methods}

Outbreak investigation and management of nosocomial transmission of chickenpox

Retrospective case finding for nosocomial transmission of chickenpox was conducted, with specific attention being paid to infection control measures and outbreak investigations between January 1, 2013 and December 31, 2017. The clinical definition of varicella is "an illness with acute onset of diffuse (generalized) papulovesicular rash without other apparent cause". All contacts in the hospital were followed up by the infection control unit staff to determine the number of susceptible contacts (non-immune to varicella) by contact tracing. Susceptible contacts were put under self-medical surveillance and requested to refrain from patient contact from Day 8 after 1st exposure to Day 21 after last exposure. Individuals were considered susceptible if they did not fulfill any one of the following criteria: 1) History of varicella; 2) Completion of varicella vaccine according to recommended schedule; 3) Positive serum VZV IgG. Postexposure prophylaxis (PEP) for susceptible contacts were administered according to CDC recommendations [2].

\section{Seroprevalence study of HCWs VZV antibodies}

A cross-sectional study on the seroprevalence rate of VZV among healthcare workers (HCWs) was conducted between January 1, 2014 and December 31, 2017 in a tertiary teaching hospital in China, with 1804 hospital staff including doctors, nurses, technicians and non-clinical workers participated in this study. The study was approved by the Ethics Committee of the hospital. Through questionnaire, information including demographic data, history of varicella and vaccination were collected from the participants. Blood samples were collected from each individual and the serum VZV antibodies were measured by enzymelinked immunosorbent assay (ELISA) using commercial kit (Beier, Beijing, China; based on manufacturer's data, the positive cutoff was defined as 0.15 if mean of absorbance of negative control $<0.05$, while $0.1+$ mean of absorbance of negative control if mean of absorbance of negative control $\geq 0.05$, with sensitivity and specificity quoted as 96.1 and $100 \%$ respectively).

Statistical analysis was performed using SPSS 20 software. Categorical variables were compared using Chisquare test. A $P$-value of less than 0.05 was considered statistically significant. Positive predictive value (PPV) and negative predictive value (NPV) of a self-reported history of varicella were calculated, with a positive serum VZV IgG considered as the gold standard of immunity against VZV.

\section{Economic evaluation of nosocomial transmission}

In order to assess the economic impact of nosocomial transmission of $\mathrm{VZV}$, two important parameters, persondays of work lost and infection control unit person-hours, were assessed. Person-days of work lost is defined as the total number of days of work loss in clinical staff for both susceptible contacts and infected cases. Infection control unit person-hours is defined as the number of hours required for infection control unit for outbreak investigation and control. The cost of performing VZV IgG ELISA was 
also calculated based on the price of equipment together with the manpower required to perform the test.

\section{Results}

\section{Outbreak investigation and control}

There were three episodes of nosocomial transmission of varicella involving 8 individuals, in which $4 \mathrm{HCWs}$ (including one doctor, one non-clinical worker and two nursing students) were infected nosocomially. Table 1 shows the characteristics of the 8 cases of varicella, and Fig. 1 shows the epidemic curve and chain of transmission in these episodes.

\section{Nosocomial transmission 1}

On May 5, 2013, a paediatrician (Case 2) presented with fever followed by generalized vesicular rash. She recalled contacting a patient (Case 1) with chickenpox on April 18, 2013 in the hospital, and she was susceptible to VZV, i.e., she was neither vaccinated nor previously infected with VZV. She was diagnosed to have varicella and requested self-isolation to prevent ongoing transmission. Contact tracing was initiated by the hospital infection control unit and a total of 23 healthcare worker contacts were identified with advice on prodromal symptoms to ensure they were not exposed to other susceptible HCWs or patients. No further new cases were identified after the above infection control measures. After this incident, serum VZV IgG testing for $\mathrm{HCW}$ was introduced by hospital health direction.

\section{Nosocomial transmission 2}

On August 22, 2013, a non-immune operator from the hospital reception center (Case 4) presented with fever, generalized vesicular rash and sore throat. She recalled one of her colleagues (Case 3), who worked with her in the same office, developed chickenpox during August 10-17, 2013. She was subsequently diagnosed to have varicella. The infection control unit was informed of the incident, with 17 healthcare worker contacts identified through contact tracing. Further history taking and laboratory testing suggested 4 of the 17 contacts were susceptible, therefore they all received post-exposure prophylaxis with oral acyclovir. No further new cases were identified after the above infection control measures.

\section{Nosocomial transmission 3}

On March 20, 2017, two nursing students (Case 5 and Case 6) from hospital outsourcing company reported to have fever and generalized vesicular rash. They worked as nursing assistants in the hospital and lived in a 8-person dormitory room. They were diagnosed to have varicella and requested self-isolation. The infection control unit was informed, with 42 healthcare worker contacts identified through contact tracing. Thorough investigation suggested 5 out of 42 contacts were susceptible. All susceptible contacts received VZV vaccination as Post-exposure Prophylaxis (PEP), with recommendation on self-isolation during incubation period provided.

Table 1 Characteristics of 8 chickenpox cases in three nosocomial transmissions, 2013-2017

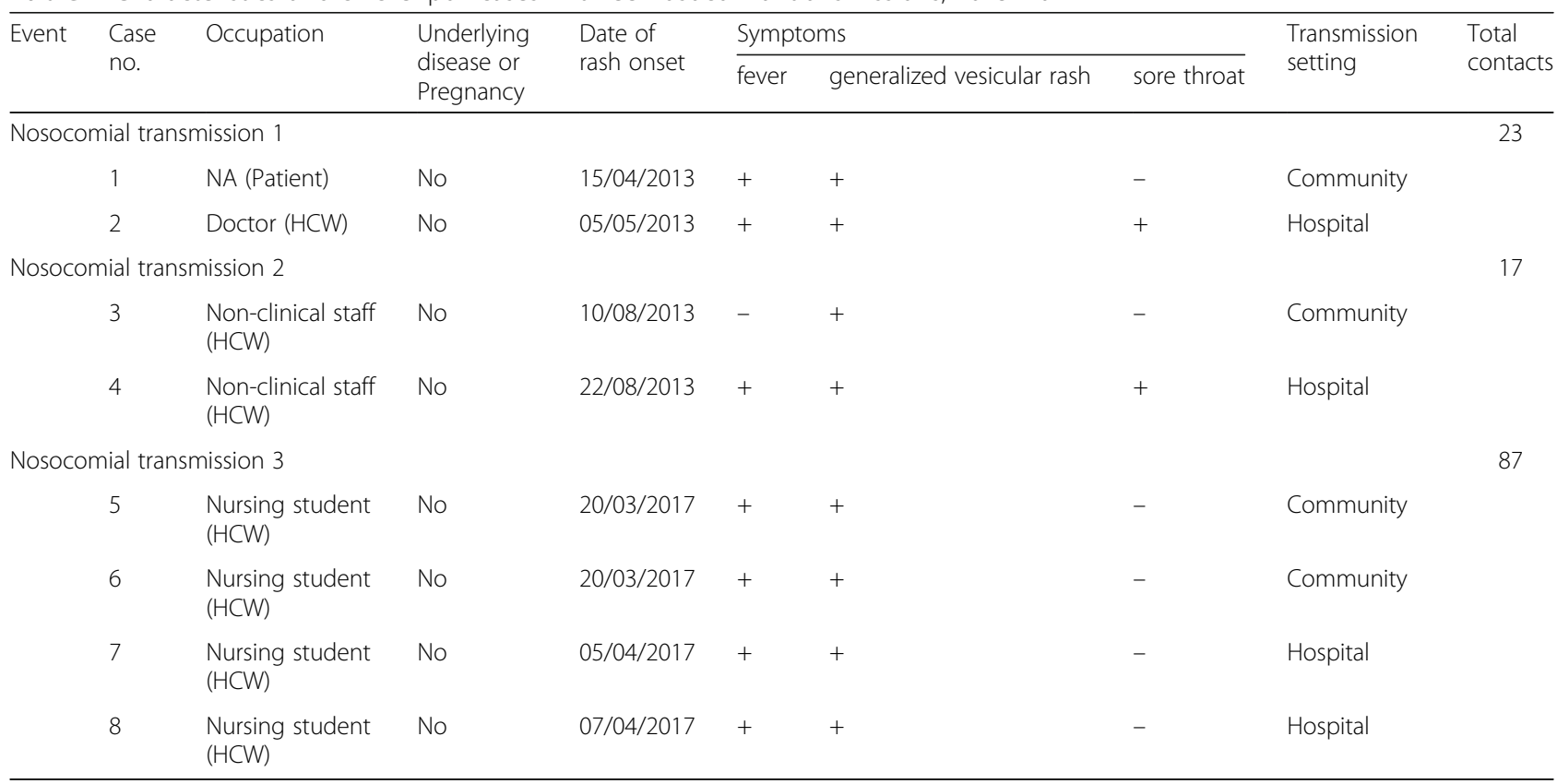




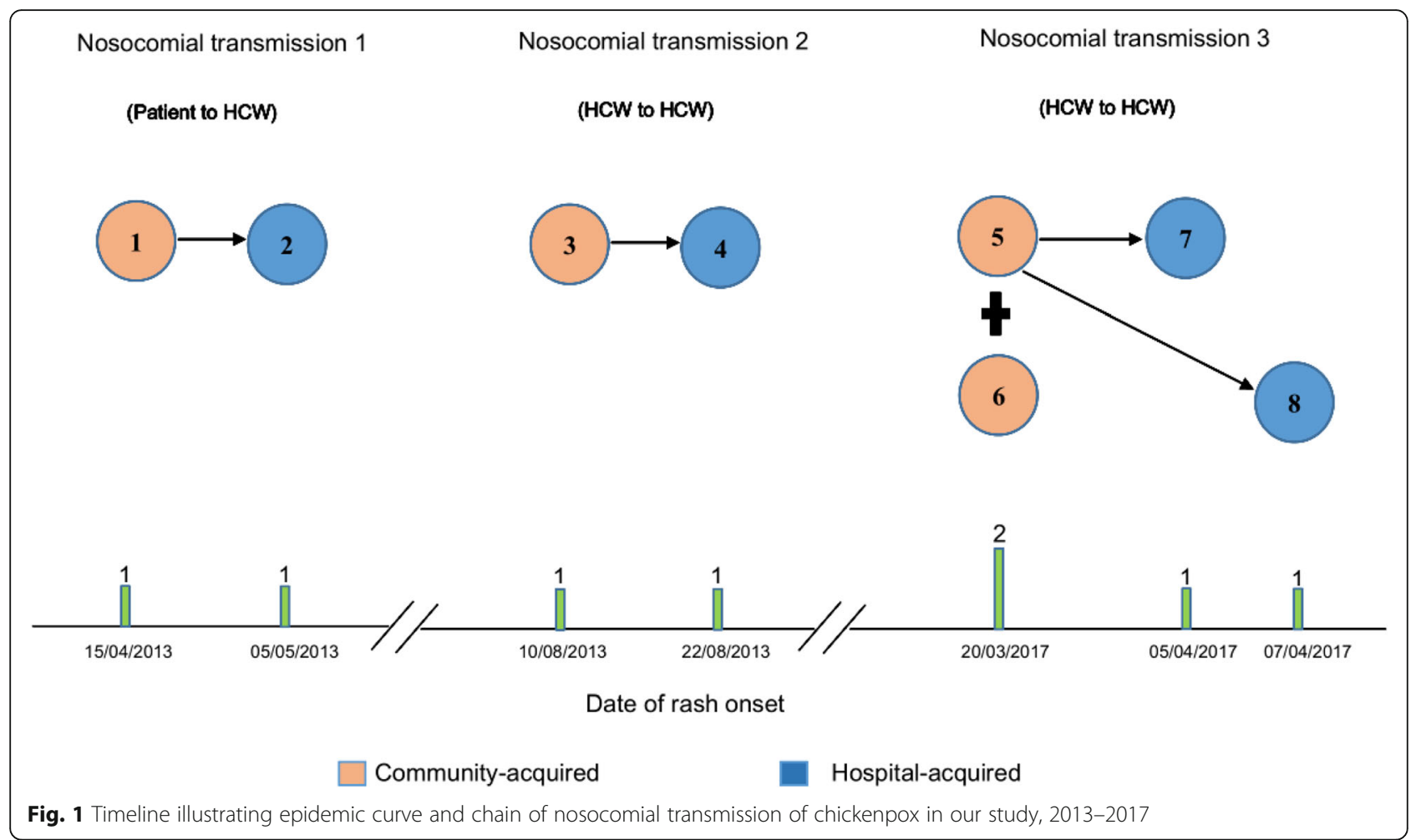

On April 5, 2017 and April 7, 2017, two nursing students (Case 7 and Case 8), who lived with Case 5 in the same dormitory, presented with similar clinical manifestations as Case 5, with VZV DNA detected from the vesicular fluid of skin lesions in both nursing students by PCR (Polymerase Chain Reaction). These two infected individuals were not classified as susceptible contacts during the first round of contact tracing, because both of them volunteered previous history of VZV vaccination. A further 45 healthcare worker contacts were identified through second round of contact tracing, and 4 out of 45 contacts were classified as susceptible individuals, with subsequent post-exposure prophylaxis provided. No further new cases were identified after the above infection control measures.

\section{Seroprevalence of VZV in HCWs}

Of the $1804 \mathrm{HCW}$ s participated, there were 215 (11.9\%) male and 1589 (88.1\%) female HCWs, with their ages

Table 2 Demographic data and VZV serostatus of 1804 healthcare workers

\begin{tabular}{llll}
\hline Variable & $\begin{array}{l}\text { Total no. } \\
(\%)\end{array}$ & \multicolumn{1}{l}{ VZV antibodies results } & \\
\cline { 4 - 4 } Gender & & Positive no.(\%) & Negative no.(\%) \\
Male & $215(11.9)$ & $186(86.5)$ & $29(13.5)$ \\
$\quad$ Female & $1589(88.1)$ & $1409(88.7)$ & $180(11.3)$ \\
Age & & & \\
$\leq 25$ & $809(44.9)$ & $689(85.2)$ & $120(14.8)$ \\
$25-35$ & $859(47.6)$ & $780(90.8)$ & $79(9.2)$ \\
$\geq 36$ & $136(7.5)$ & $126(92.6)$ & $10(7.4)$ \\
Occupation & & $133(86.9)$ & $20(13.1)$ \\
Physician & $153(8.5)$ & $1097(88.6)$ & $141(11.4)$ \\
Nurse & $1238(68.6)$ & $190(86.0)$ & $31(14.0)$ \\
Technician & $221(12.3)$ & $175(91.1)$ & $17(8.9)$ \\
Non-clinical workers & $192(10.6)$ & $1595(88.4 \%)$ & $209(11.6 \%)$ \\
Total & 1804 & & 0.382 \\
\hline
\end{tabular}


ranging from 17 to 60 years (median age: 27 years). Table 2 shows the demographic characteristics of the participated HCWs. The overall seroprevalence rate of VZV was $88.4 \%$. The seroprevalence rates of HCWs with different genders and occupations showed no statistically significant differences. HCWs aged $\geq 36$ years were found to have the highest seroprevalence rate $(92.6 \%)$, while HCWs aged $\leq 25$ years were found to have the lowest seroprevalence rate $(85.2 \%)$.

Table 3 shows the positive and negative predictive values of a self-reported history of varicella, with a positive serum VZV IgG considered as the gold standard of immunity against VZV. Of the 1804 participants, 214 (11.9\%) HCWs reported history of varicella, with 173 (80.8\%) tested positive and 41 (19.2\%) tested negative for serum VZV IgG; while 1590 (88.1\%) HCWs reported no history of varicella, with $1422(89.4 \%)$ tested positive and $168(10.6 \%)$ tested negative for serum VZV IgG. The PPV and NPV of a self-reported history of varicella were 80.8 and $10.6 \%$ respectively.

\section{Economic evaluation of nosocomial transmission}

A total of 20 person-days of work were lost by infected clinical staff during the above episodes, based on the fact that immunocompetent persons are unlikely to be infectious after day 5 of rash. Isolating susceptible healthcare worker contacts from duty from days 8 to 21 after last exposure would result in further working hours lost, with 470 person-hours loss for the above three episodes. Therefore, a total of 490 person-hours were lost during the above described three transmissions, with an estimate of 163.3 person-hours being lost per nosocomial transmission.

During the first two transmissions, infection control unit required $40 \mathrm{~h}$ per transmission to contact trace, follow up and provide counseling to all involved healthcare workers. As the third transmission involved more clinical staff, infection control unit required $180 \mathrm{~h}$ for infection control measures. Therefore, a total of 260 infection control unit person-hours were required for the three transmissions, with 86.7 infection control unit personhours required per transmission.

The cost of each VZV IgG ELISA commercial kit is 11 RMB (equivalent to 1.59 USD), and manpower for performing each ELISA kit is 2.5RMB (equivalent to 0.36

Table 3 VZV serological test results and history of varicella among 1804 HCWs

\begin{tabular}{lcllll}
\hline History of & \multicolumn{1}{l}{ VZV antibody results } & & & \\
\cline { 2 - 6 } Varicella & Total (no.) & Positive no.(\%) & Negative no.(\%) & PPV & NPV \\
\hline Positive & 214 & $173(80.8)$ & $41(19.2)$ & 80.8 & 10.6 \\
Negative $^{a}$ & 1590 & $1422(89.4)$ & $168(10.6)$ & & \\
\hline
\end{tabular}

${ }_{\text {ancluding uncertain history }}$
USD) in our laboratory. With a total of 127 tests performed as a result of nosocomial transmissions, the total cost of VZV IgG ELISA screening is 1714.5 RMB (around 248 USD), with around 83 USD per nosocomial transmission.

\section{Discussion}

Varicella zoster virus (VZV) is a highly contagious herpesvirus with potential for nosocomial transmission, with the infectious period starting 2 days before the onset of rash, and lasting for 5-7 days after the appearance of rash [9-11]. Several studies have demonstrated varicella can be transmitted to susceptible individuals through airborne route $[12,13]$. Susceptible individuals can acquire primary infection from contacting patients with dermatomal zoster [12-18]. It is shown in our study that nosocomial transmission is possible.

Nosocomial outbreaks of varicella involving both healthcare workers and patients are reported in other countries [5, 19-22]. Common measures for prevention of varicella transmission in the hospital setting include rapid isolation of patient in negative pressure room together with administration of post-exposure prophylaxis to susceptible individuals in the form of varicella zoster vaccine, varicella zoster immunoglobulin, and oral acyclovir. Majority of the studies showed difficulty in prevention of secondary cases after an index case of varicella was identified in the hospital. Common difficulties encountered include improper isolation facilities and non-specific symptoms during initial presentation, especially when the infectious period of VZV infection starts 2 days before the onset of rash [5]. Furthermore, secondary cases still occurred with post-exposure prophylaxis despite it was given within timeframe [20]. Studies in developing countries such as Indonesia also encountered the problem of lack of supply of varicella zoster immunoglobulin [22]. With the multiple difficulties encountered from previous studies and our experience, routine screening of serum VZV IgG in healthcare workers might be required to reduce the chance of nosocomial transmission in the future.

The seroprevalence rate of VZV in healthcare workers varies according to different geographical areas, with the prevalence of seronegativity ranging from $<5 \%$ in the USA, $8.9 \%$ in Taiwan, $19 \%$ in Saudi Arabia, 26\% in India to approximately $50 \%$ in Sri Lanka [23, 24]. In our study, the serum VZV antibodies of 1804 HCWs were measured with $88.4 \%$ found to be seropositive. The results showed that a considerable proportion (11.6\%) of HCWs were still susceptible to varicella, and HCWs can be a potential source of nosocomial transmission, indicating the importance of the screening of serum VZV IgG and vaccination of susceptible $\mathrm{HCWs}$, as exemplified by the three episodes of nosocomial transmissions documented in our hospital in the past 5 years. All the $4 \mathrm{HCWs}$ 
involved were not screened for serum VZV IgG prior to employment, as a result, they were involved in the three episodes of nosocomial transmission. Nosocomial VZV infection has great economic impact, as laboratory testing, contact tracing and self-isolation of HCWs could represent both an increase in laboratory cost and a decrease in manpower. A reasonable estimate of 163.3 person-days of work were lost as a result of each nosocomial transmission and 86.7 infection control unit person-hours were required during each outbreak investigation and control, together with an estimated laboratory cost of 83 USD per nosocomial transmission, depending on the size of the outbreak.

In our study, the seroprevalence rate of VZV in HCWs significantly increased with age, and this could be explained by accumulated time of exposure to VZV during lifetime in elderly. The seroprevalence rates of HCWs with different genders and occupations showed no statistically significant differences.

In order to investigate whether self-reported history could replace serum VZV IgG screening in HCWs, selfreported history of varicella in HCWs was compared with the serum VZV IgG result to assess the reliability of self-reported history. Our study suggested that a positive history of varicella provided by HCWs is not a good predictor of positive serum VZV IgG, as our study demonstrated a false positive rate of $19.2 \%$. On the other hand, $89.4 \%$ of HCWs with a negative history of varicella turned out to be seropositive, suggesting negative selfreported history could not rule out existing immunity towards VZV. Review of literature from different countries suggested a self-reported history of varicella has a positive predictive value of $92.5-99.5 \%$ and a negative predictive value of $2.5-14.4 \%$ [24-27]. Comparison of the above data suggested that the negative predictive value from our study is similar to that reported in the literature. However, the positive predictive value is only $80.8 \%$ in our study. As natural varicella usually occurred during childhood, healthcare workers could only rely on their parents for the history of varicella. One possible postulation for the above discrepancy could be due to the lower level of education in the past in China, as it may be difficult for parents to distinguish between varicella and other febrile exanthematous diseases, leading to lower positive predictive value in this case. Hence, self-reported history of varicella is not a reliable predictor of VZV immunity in our locality, therefore screening of serum VZV IgG for all HCWs is indicated, and susceptible workers should be vaccinated so as to prevent nosocomial transmission of varicella.

The limitation of our study is that not all HCWs participated in our study, as $244 \mathrm{HCW}$ refused screening of serum VZV IgG, which may in turn lead to selection bias. However, further statistical analysis did not reveal any statistical differences by chi-square test between these HCWs and those who participated. Furthermore, this present study included a large number of HCWs with a wide age range and diverse occupations, therefore it still could provide a convincing approximation of the situation of VZV immunity in HCWs in China.

With the advancement in the field of transplantation and medicine, we are encountering more immunocompromised patients in our daily practice. Our study clearly demonstrated that assuming seropositivity based on selfreported history of varicella is unreliable, and significant proportion (10.6\%) of existing HCWs are still susceptible to VZV. Further intervention should be implemented in the health care system to prevent hospital transmission of varicella. It is time for us to implement routine serum VZV IgG testing and immunization of HCWs if serum VZV IgG result turns out to be negative, not only to reduce the cost of outbreak investigation, but more importantly to ensure safety of both HCWs and our patients. Such intervention should not only be performed on newly recruited members, but also to existing healthcare workers in the hospital.

\section{Conclusions}

Nosocomial transmission of VZV occurred repeatedly in the hospital setting. An alarming 11.6\% of HCWs were seronegative for $\mathrm{VZV}$, which might increase the risk of nosocomial infection and outbreak for other susceptible co-workers and patients. This is especially important in the setting of a teaching hospital where many immunocompromised patients were managed. Furthermore, the positive predictive value of self-reported varicella on seroprevalence rate in our study was lower than those reported in other countries, therefore serological testing of VZV antibodies with subsequent vaccination for all non-immune HCWs should be considered.

\section{Abbreviations \\ CDC: Centers for Disease Control and Prevention; ELISA: Enzyme-linked immunosorbent assay; FAMA: Fluorescent antibody to membrane antigen; HCWs: Healthcare workers; NPV: Negative predictive value; PCR: Polymerase Chain Reaction; PEP: Post-exposure prophylaxis; PPV: Positive predictive value; VZV: Varicella zoster virus}

\section{Acknowledgements}

Not applicable.

\section{Authors' contributions}

$J Y, J L, F X, H Y, J F-W C$ and K-YY contributed to the design of the study. JY, GD and ML participated in the acquisition and analysis of data. SK-FL and RW-TL performed the laboratory tests. JY drafted the manuscript. KH-YC, JF-WC and $\mathrm{K}-\mathrm{YY}$ revised the manuscript. All of the authors read and approved the final draft of the manuscript

\section{Funding}

This study was partly supported by the High Level Hospital-Summit Program in Guangdong, The University of Hong Kong-Shenzhen Hospital; the Sanming Project of Medicine in Shenzhen, and the Collaborative Innovation Center for Diagnosis and Treatment of Infectious Diseases, the Ministry of Education of China. The sponsors had no role in the design and conduct of 
the study, in the collection, analysis and interpretation of data, or in the preparation, review or approval of the manuscript.

\section{Availability of data and materials}

The datasets used and analysed during the current study available from the corresponding author on reasonable request.

\section{Ethics approval and consent to participate}

The study was approved and access of raw data was granted by the Ethics Committee of the University of Hong Kong-Shenzhen Hospital. Because the study was retrospective without any interventions, the requirement for informed consent was waived.

\section{Consent for publication}

Not applicable.

\section{Competing interests}

The authors declare that they have no competing interests.

\section{Author details}

'Department of Clinical Microbiology and Infection Control, The University of Hong Kong - Shenzhen Hospital, Shenzhen, China. ${ }^{2}$ State Key Laboratory of Emerging Infectious Diseases, The University of Hong Kong, Pokfulam, Hong Kong, Special Administrative Region of China. ${ }^{3}$ Department of Microbiology, Li Ka Shing Faculty of Medicine, The University of Hong Kong, Pokfulam, Hong Kong, Special Administrative Region of China. ${ }^{4}$ Carol Yu Centre for Infection, Li Ka Shing Faculty of Medicine, The University of Hong Kong, Pokfulam, Hong Kong, Special Administrative Region of China. ${ }^{5}$ The Collaborative Innovation Center for Diagnosis and Treatment of Infectious Diseases, The University of Hong Kong, Pokfulam, Hong Kong, Special Administrative Region of China.

Received: 16 January 2019 Accepted: 26 June 2019

Published online: 05 July 2019

\section{References}

1. Straus SE, Ostrove JM, Inchauspé G, Felser JM, Freifeld A, Croen KD, et al. $\mathrm{NIH}$ conference. Varicella-zoster virus infections. Biology, natural history, treatment, and prevention. Ann Intern Med. 1988;108(2):221-37.

2. Gabutti G, Franchi M, Maniscalco L, Stefanati A. Varicella-Zoster virus: pathogenesis, incidence patterns and vaccination programs. Minerva Pediatr. 2016;68(3):213-25

3. Freer G, Pistello M. Varicella-zoster virus infection: natural history, clinical manifestations, immunity and current and future vaccination strategies. New Microbiol. 2018;41(2):95-105.

4. Marin M, Güris D, Chaves SS, Schmid S, Seward JF. Centers for Disease Control and Prevention. Prevention of varicella: recommendations of the advisory committee on immunization practices (ACIP). MMWR Recomm Rep. 2007;56(RR-4):1-40.

5. Aly NY, Al Obaid I, Al-Qulooshi N, Zahed Z. Occupationally related outbreak of chickenpox in an intensive care unit. Med Princ Pract. 2007;16(5):399-401.

6. Antela A, Fortún J, Navas E, Hermida JM, Fernández-Muñoz R, Guerrero A. Nosocomial varicella: study of an epidemic outbreak among immunosuppressed patients. Enferm Infecc Microbiol Clin. 1991;9(6):357-60.

7. Chin TL, MacGowan AP, Jacobson SK, Donati M. Viral infections in pregnancy: advice for healthcare workers. J Hosp Infect. 2014;87(1):11-24. https://doi.org/10.1016/j.jhin.2013.12.011.

8. World Health Organization. The immunological basis for immunization series: module 10: Varicella-zoster virus. Geneva: World Health Organization; 2008.

9. Heininger U, Seward JF. Varicella. Lancet. 2006;368(9544):1365-76.

10. Gershon AA, Breuer J, Cohen Jl, Cohrs RJ, Gershon MD, Gilden D, et al. Varicella zoster virus infection. Nat Rev Dis Primers. 2015;1:15016. https://doi. org/10.1038/nrdp.2015.16.

11. Ouwendijk WJ, Verjans GM. Pathogenesis of varicelloviruses in primates. J Pathol. 2015;235:298-311. https://doi.org/10.1002/path.4451.

12. Gustafson TL, Lavely GB, Brawner ER Jr, Hutcheson RH Jr, Wright PF, Schaffner $W$. An outbreak of airborne nosocomial varicella. Pediatrics. 1982;70:550-6.

13. Leclair JM, Zaia JA, Levin MJ, Congdon RG, Goldmann DA. Airborne transmission of chickenpox in a hospital. N Engl J Med. 1980;302:450-3.
14. Lopez AS, Burnett-Hartman A, Nambiar R, Ritz L, Owens P, Loparev VN, et al. Transmission of a newly characterized strain of varicella-zoster virus from a patient with herpes zoster in a long-term-care facility, West Virginia, 2004. J Infect Dis. 2008;197:646-53. https://doi.org/10.1086/527419.

15. Bloch KC, Johnson JG. Varicella zoster virus transmission in the vaccine era: unmasking the role of herpes zoster. J Infect Dis. 2012;205:1331-3. https:// doi.org/10.1093/infdis/jis214.

16. Saidel-Odes L, Borer A, Riesenberg K, Frenkel A, Sherlis R, Bouhnick L, et al. An outbreak of varicella in staff nurses exposed to a patient with localized herpes zoster. Scand J Infect Dis. 2010;42:620-2. https://doi.org/10.3109/ 00365541003754436

17. Viner K, Perella D, Lopez A, Bialek S, Newbern C, Pierre R, et al. Transmission of varicella zoster virus from individuals with herpes zoster or varicella in school and day care settings. J Infect Dis. 2012;205:1336-41. https://doi.org/ 10.1093/infdis/jis207.

18. Cholongitas E, llonidis G. Transmission of varicella-zoster virus originating from a patient with localized herpes zoster: implications for infection control? Am J Infect Control. 2010;38:669-70. https://doi.org/10.1016/j.ajic. 2010.02.010.

19. Okamoto A, Abe A, Okamoto M, Kobayashi T, Inaguma Y, Tokuda M, et al. A varicella outbreak in B-cell lymphoma patients receiving rituximabcontaining chemotherapy. J Infect Chemother. 2014 Dec;20(12):774-7. https://doi.org/10.1016/j.jiac.2014.08.006

20. Sood S. Occupationally related outbreak of chickenpox in hospital staff: a learning experience. J Clin Diagn Res. 2013 Oct;7(10):2294-5. https://doi.org/ 10.7860/JCDR/2013/5780.3502

21. Kellie SM, Makvandi M, Muller ML. Management and outcome of a varicella exposure in a neonatal intensive care unit: lessons for the vaccine era. Am J Infect Control. 2011 Dec;39(10):844-8. https://doi.org/ 10.1016/j.ajic.2011.02.006

22. Gunawan S, Linardi P, Tawaluyan K, Mantik MF, Veerman AJ. Varicella outbreak in a pediatric oncology Ward: the Manado experience. Asian Pac J Cancer Prev. 2010;11(2):289-92.

23. World Health Organization. Varicella and herpes zoster vaccines: WHO position paper. June 2014. Wkly Epidemiol Rec. 2014;89(25):265-87.

24. Wu MF, Yang YW, Lin WY, Chang CY, Soon MS, Liu CE. Varicella zoster virus infection among healthcare workers in Taiwan: seroprevalence and predictive value of history of varicella infection. J Hosp Infect. 2012 Feb; 80(2):162-7. https://doi.org/10.1016/j.jhin.2011.11.011.

25. Kang JH, Park YS, Park SY, Kim SB, Ko KP, Seo YH. Varicella seroprevalence among health care workers in Korea: validity of self-reported history and cost-effectiveness of prevaccination screening. Am J Infect Control. 2014 Aug;42(8):885-7. https://doi.org/10.1016/j.ajic.2014.05.013.

26. De Juanes JR, Gil A, San-Martín M, González A, Esteban J, García de Codes A Seroprevalence of varicella antibodies in healthcare workers and health sciences students reliability of self-reported history of varicella. Vaccine. 2005;23(12):1434-6.

27. Kumakura S, Onoda K, Hirose M. Self-reported histories of disease and vaccination against measles, mumps, rubella and varicella in health care personnel in Japan. J Infect Chemother. 2014 Mar;20(3):194-8. https://doi. org/10.1016/j.jiac.2013.10.006

\section{Publisher's Note}

Springer Nature remains neutral with regard to jurisdictional claims in published maps and institutional affiliations.

Ready to submit your research? Choose BMC and benefit from

- fast, convenient online submission

- thorough peer review by experienced researchers in your field

- rapid publication on acceptance

- support for research data, including large and complex data types

- gold Open Access which fosters wider collaboration and increased citations

- maximum visibility for your research: over $100 \mathrm{M}$ website views per year

At BMC, research is always in progress.

Learn more biomedcentral.com/submissions 